Cite this: DOI: $00.0000 / \operatorname{xxxxxxxxxx}$

Received Date

Accepted Date

DOI: 00.0000/xxxxxxxxxx

\section{Tuning the Dielectric Response in a NC Material through Nanoparticle Morphology}

\author{
Archita Adluri, ${ }^{a}$ Brett Henderson ${ }^{a}$ and Irina Paci ${ }^{*}{ }^{\ddagger}$
}

\begin{abstract}
The introduction of metal cluster dopants and molecular-scale inclusions in metal oxide matrices provides an opportunity for exploring new high- $k$ solid-state dielectrics with tunable response. The quantum properties of molecular nanoparticles depend strongly on their size and shape, a characteristic that can be exploited in changing the response properties of a material, while the small nanoparticle size can help limit the usual issues of conduction and leakage. Here, we model the polarization of molecular-scale silver inclusions in magnesium oxide, using the Modern Theory of Polarization and Car-Parinello Molecular Dynamics (CPMD). Several trends are considered, including nanoparticle size, shape and orientation relative to the applied field. Dielectric permittivity enhancements of $30-100 \%$ were observed with inclusion sizes varying from 8 to 32 atoms, considering both rod-like and disk-like inclusions, with alignment either parallel or perpendicular to the external field. Currents calculated using the modern theory of polarization with periodic boundary conditions can experience box edge jumps due to the distortion of the matrix during the simulations - an approach for addressing these issues in CPMD calculations is outlined within.
\end{abstract}

The demand for new high- $\kappa$ dielectric materials is increasing in a world where energy storage and processing power demands are reaching the limit of traditional single-phase materials 1 . Nanocomposite (NC) materials that combine a standard dielectric matrix with inclusions of high and mid- $\kappa$, perhaps even conducting materials, have been advanced as a possible avenue for the expansion of the possible range of materials available for device engineering, due to their highly tunable dielectric and mechanical properties. $2[3$

One area in need of innovation is that of materials with tunable dielectric constant $(\kappa)$ for electrical and computational devices that rely on metal-oxide semiconducting materials such as transistors, high-density charge storage super-capacitors, and emerging technologies in quantum computing. $\frac{4}{8}$ Advancements in semiconductor manufacturing have lead to increased miniaturization and densification of electrical circuits allowing for increasing computing power or more efficient charge storage. As traditional single phase $\mathrm{Si} / \mathrm{SiO}_{2}$ gate manufacturing became more precise, we were able to make smaller transistors and more efficient capacitors. However as processor chips approach

${ }^{a}$ Department of Chemistry and the Centre for Advanced Materials and Related Technologies, University of Victoria, PO Box 1700, 3800 Finnerty Road, Victoria, BC, V8W 2Y2, Canada.

† Email: ipaci@uvic.ca. few-nanometer sizes, significant current leakage and tunnelling effects often dominate the ability of a dielectric material to hold an electrical charge.

Traditional $\mathrm{Si} / \mathrm{SiO}_{2}$ has thus given way to more complex, higher-permittivity materials such as silicon-germanium and hafnium oxides ${ }^{9}$, nitrogen-doping 10 , polymer enriched metaloxides 11 and other multi-phase materials in order to address these issues. Molecular nanoparticle of various sizes and shapes, 2D sheet-type dopants such as graphene sheets, metal and halogen doping and mixed-metal oxides have all been considered

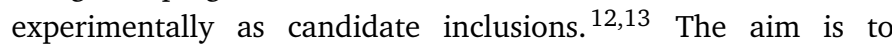
selectively modify the permittivity (dielectric constant $\kappa$ ), and therefore the charge capacity of the resulting material. The research so far has shown that at the atomic scale, even a point defect or a single atom defect leads to noticeable changes in polarization potential of a material. $14 \mid 15$

Metallic nanoparticles in the molecular regime (nanoparticles with up to 100 or so atoms) bridge the gap between single-atom dopants in classical semiconductors and traditional nanoparticles with "metallic properties", while providing a range of unique features.16 Molecular nanoparticles (mNPs) are insulating, do not present a band structure and the high-electron density of traditional nanoparticles, which often leads to low percolation 
thresholds and tunnelling. mNPs are essentially a source of highly polarizable electron density, their quantum behaviour requiring quantum mechanical treatments to model their contribution to local polarization effects.

Theoretical approaches for predicting the response of mixed materials to applied fields and for optimizing their morphology and elemental composition have been developed, although several challenges remain. Effective medium approximations ${ }^{17-19}$ are suitable for macroscale mixtures, especially where experimental data is broadly available. Classical atomistic approaches work at the nanoscale as well as for certain optical responses, but do not encompass, or provide insight into, the quantum response and local field effects that can dominate the dielectric response in complex-structured solid-state materials. $\frac{20+22}{2}$ Direct DFT approaches have been used for doped materials, but a complete treatment of the quantum polarization of the mixed material has not been attempted until recently. ${ }^{1]}$

In a previous publication from our group, $\frac{1}{1}$ we used the modern theory of polarization ${ }^{2324}$ to develop a model for describing the polarization of mNP NCs. We sought to unravel the contributions of the inclusion and matrix to the overall response of a model $\mathrm{Ag}_{8} / \mathrm{MgO} \mathrm{NC}$ and found significant interfacial effects between the NC and matrix produced greater than expected polarization response. Here, we follow up on that initial study, to explore the effects of the distribution and shape of the mNP inclusions on the dielectric capacity of a metal-oxide material. We find that at mNP scales of $<30$ atoms the size, shape and orientation of the inclusion greatly alter how the inclusion electrons behave and therefore affect the atomic-scale polarization. This is important to understand as we make smaller and more precise dielectric materials.

\section{Theory and Model System}

\subsection{Models}

The NC systems used for this study were based on a magnesium oxide matrix with an array of silver mNPs inclusions. $\mathrm{MgO}$ is a well researched high- $\kappa$ metal-oxide gate dielectric ${ }^{25 \mid 26}$ and is used in spintronics. $\frac{2728}{} \mathrm{An}$ inert material with a bulk dielectric constant of 8-9.8 and a bandgap of $7.8 \mathrm{eV}, \mathrm{MgO}$ is one of the most convenient crystalline oxides from a computational standpoint, due to its cubic crystal structure and isotropic polarization, (unlike $\mathrm{SiO}_{2}$ which can present anisotropic polarization depending on its structure), and a low number of polarizable d-electrons (unlike other mid/high- $\kappa$ materials, such as $\mathrm{HfO}_{2}$ ). This is especially important in Car-Parinello Molecular dynamics, where valence electrons in matrix atoms greatly increase the computational cost. Models were built using the Avogadro 29 software. An example $\mathrm{NC}$ with a $\mathrm{Ag}_{12}$ inclusion is shown in 1 below.

Three rod-like inclusions and five disk-like inclusions were considered, as shown in Figures 2 and 3 Inclusions were built on an ideal Ag fcc lattice, but allowed to optimize in the CPMD simula-

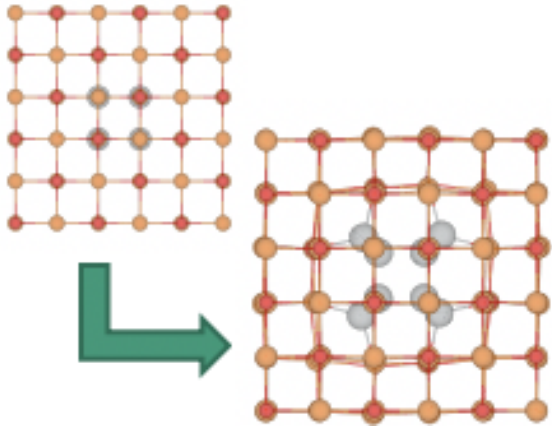

Fig. 1 The unit cell of the $\mathrm{Ag}_{12} / \mathrm{MgO} \mathrm{NC}$, before and after geometry optimization. $\mathrm{Mg}^{2+}, \mathrm{O}^{2-}$ and $\mathrm{Ag}$ are shown in orange, red and grey, respectively.

tion. The matrix extended $2 \mathrm{Mg}$-O layers beyond the edge of the inclusion in each direction. This kept the cell from conducting into its periodic image while keeping control of loading and cell size, and ensuring appropriate lattice continuation at boundaries.

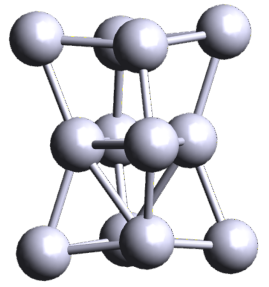

(a) $223 \mathrm{ROD}$

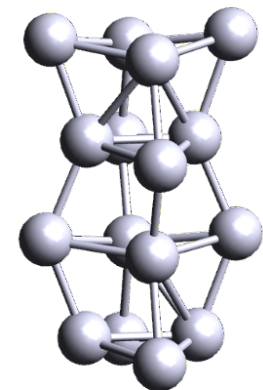

(b) 224 ROD

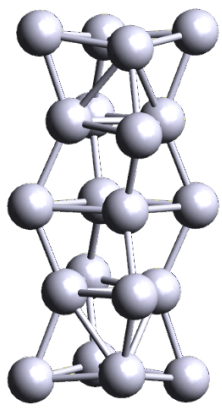

(c) 225 ROD
Fig. 2 Rod-shaped nanoparticles used in this work. The nanoparticles were named for the number of repeating units in the $x, y$, and $z$ directions, and include 12, 16 and 20 atoms for panels (a), (b) and (c), respectively.

\subsection{Low-frequency Ionic and Electronic polarization}

The process for calculating the low-frequency polarization of a NC material using the modern theory of polarization, as well as our methodology of partitioning the complex material's response, are described in detail in Ref. ${ }^{1}$. Briefly, the CPMD method is used in the Quantum Espresso (QE) code, ${ }^{30 \mid 31}$ with the two major stages of the calculation, structural structure relaxation and polarization, outlined in the following two subsections.

\subsubsection{Electronic Structure Calculations}

The relaxed atomic and electronic structure was found using CPMD, $\stackrel{30131}{ }$ in a series of optimization steps. Initial electron minimization calculated with Gram-Schmidt orthogonalization, was followed by a series of damped dynamics steps to allow both ions and electrons to minimize fully, and an additional step to relax lattice vectors to remove any additional stress on the cell due to presence of the inclusion within the matrix. These are Steps 1-6 shown in Figure 4 


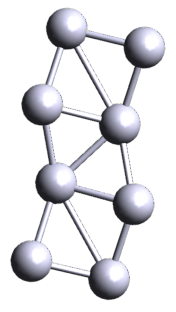

(a) 241 DISK

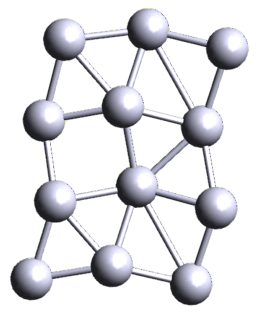

(b) 341 DISK

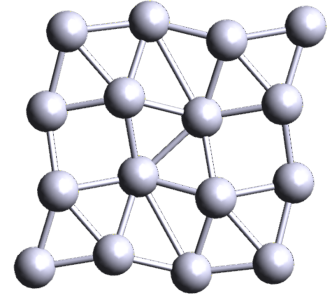

(c) 441 DISK

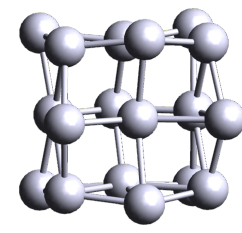

(d) 332 DISK

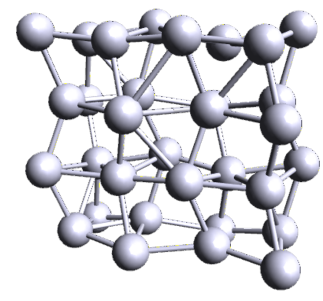

(e) 442 DISK
Fig. 3 Disk-shaped molecular nanoparticles used in this work. The nanoparticles were named for the number of repeating units in the $x$, $y$, and $z$ directions, and include 8, 12, 16, 18 and 32 atoms for panels (a)-(e), respectively.

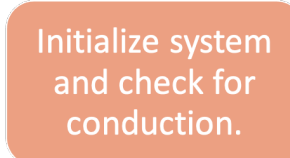

Minimize quickly not accurately

Fine tuning electron positions
- Step 1: Initial electron minimization Gram-Schmidt orthogonalization.

- Step 2: Bring all electrons to the ground state to get initial ion configuration.

- Step 3: Steepest descent minimization.

- Step 4: Damped ion positions slightly to allow for minor adjustments in ion position.

\section{- Step 5: Further relaxation of cell stress.}

- Step 6: Proper electronic structure calculation.
Fig. 4 Structure calculation steps using QuantumEspresso.

\subsubsection{Polarization}

The modern theory of polarization allows calculation of the polarization change of a material upon application of a finite electric field. The low frequency dielectric constant $\left(\varepsilon_{\mathrm{r}}\right)$ is calculated according to Equation 13233

$$
\varepsilon_{\mathrm{r}}=1+\frac{4 \pi\left(P_{\text {damped }}^{\varepsilon}-P_{\text {relax }}^{\varepsilon}\right)}{\Omega \varepsilon_{\text {field }}}
$$

In $\mathrm{QE}$, this was achieved using static field $\left(\varepsilon_{\text {field }}\right)$ of 0.001 a.u applied along the z-axis. The reference polarization is that of the cell without an applied field. The electronic and ionic polarization is calculated using damped dynamics ( $P_{\text {damped }}^{\varepsilon}$ ) shows mainly ionic polarization. After fully relaxing the electronic structure in the field $\left(P_{\text {relax }}^{\varepsilon}\right)$ describes electronic polarization as the electrons are allowed to polarize and the Ag electron density is allowed to move into the $\mathrm{Ag}-\mathrm{MgO}$ interface. The values calculated for $\varepsilon_{\mathrm{r}}$ are not 'true' dielectric constants for a real material in that surface charge and edge effects are not taken into account as the material simulated is periodic and the value is a volume average. Dielectric constants in real materials are volume and \%loading dependent, so for increased accuracy \%loading ratios of inclusions to matrix were kept between 3-5\% while still allowing sufficient matrix to separate periodic images of the mNP. Here, the \%loading is reported in numbers of atoms, as the volume occupied by an inclusion atom is, on average, the same as the volume occupied by individual matrix ions (as shown in our previous work ${ }^{1}$ ).

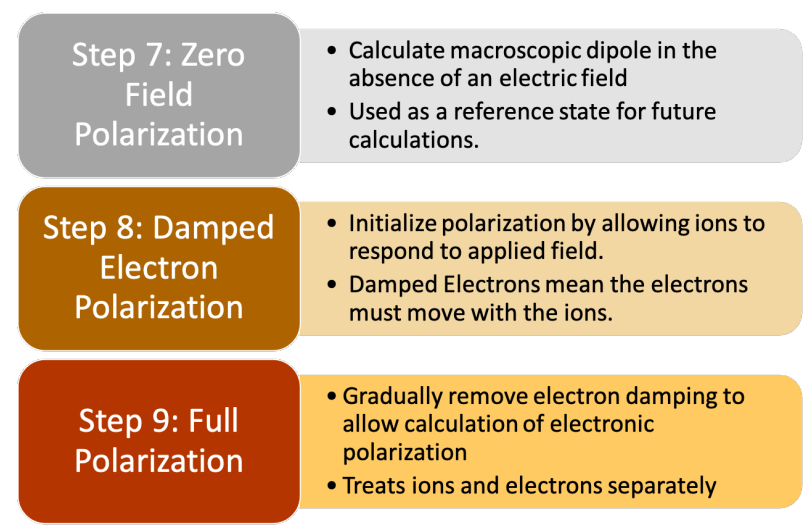

Fig. 5 Polarization steps using QuantumEspresso - step counting continued from electronic structure calculation.

\subsubsection{Quantum Of Polarization}

The explicit 'particle-like' electrons in CPMD will occasionally polarize into the neighboring image cell (or, more specifically, to the other side of the simulation box), shifting the charge distribution relative to the 'reference' coordinate system in which the polarization is calculated. The reported polarization then shifts (positive or negative) in value equal to nxboxlength. This behaviour is equivalent to the creation of quantum of polarization effects described by Spaldin 24 . The calculated polarization of any periodic system is multiply defined, and the polarization of electrons, in our case, leads to different electron distributions over the ions in the zero-field and polarized states. This is equivalent to a distortion of the simulation cell, as discussed by Spaldin, or alternately a change of the reference state for polarization calculation. As the matrix is deformed by larger and un-symmetrical inclusions, the more polarization corrections were needed.

Therefore, care must be taken in processing the charge distribution when calculating the effective polarization, particularly for low-symmetry systems or deformed crystal lattices. Our previous model ${ }^{1}$ did not suffer effects of distortion of polarization due to high symmetry and small size of inclusion. The mathematical corrections for these polarization jumps during post-processing are shown in Figure 6 .

\subsection{Born Effective Charge}

To understand the electronic polarization effects in the composite, we examine its Born effective charge tensor $\left(Z_{i j}^{*}\right.$, BEC). BECs 

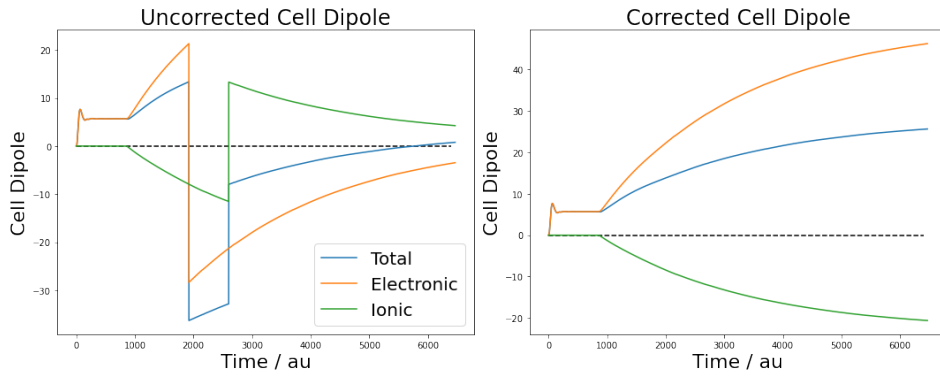

Fig. 6 Mathematical correction of polarization values due to quantum of polarization effect

reflect the changes in the local charge distribution of the material as a result of ionic displacements or an applied electric field. The $\mathrm{BEC}$ tensor relates the change in polarization due to the movement of an atom. More specifically it allows us to look at the movement of electrons/electron density in the (z or k?) direction with ion nuclei clamped due to the application of an electrical field. Eq. 2 shows the general formula for BECs:

$$
Z_{i j}^{*}=-e \frac{\partial F_{i}}{\partial E_{j}}
$$

In a bulk single material, the static charges of constituent elements (or ions) are their nominal valence charge ${ }^{34}$ ( +2 for oxygen, -2 for magnesium in the case of $\mathrm{MgO}$ ). Upon application of an electrical field, the average overall BEC tensor remains the same as all the atoms experience the field uniformly. In a NC, however, significant changes in the charge distribution of the matrix ions arise from the influence of mNP electrons, particularly under an applied field. This occurs particularly in the ions close to the mNP-matrix interface, where the microscopic 'induced field' from the moving Ag valence electrons is strongest. BEC maps are thus a useful tool for mapping the local charge density and spatial manipulation of induced fields at such small scales. Here, we calculated the difference in force experienced by each atom under zero-field and static polarization. Forces were normalized to the bulk matrix to highlight the effect of the NP.

\section{Numerical Details}

Electronic structure calculations were carried out in a zero field environment. Electronic structure, the relaxed crystal structures and polarization results were calculated using the CPMD methodology implemented in the Quantum Espresso code version 6.0 and 6.6, using a plane wave-basis set built using LDA functional. $\mathrm{Mg}$ atoms described using Bachelet-Hamann-Schluter ${ }^{35}$ norm-conserving pseudopotentials, and Rappe-Rabe-Kaxiras-Joannopoulos ${ }^{36}$ ultra-soft pseudopotentials from MaterialsCloud ${ }^{37}$ were used for $\mathrm{O}$ and Ag. The total energy convergence criteria was increased from the standard $10^{-4}$ to $10^{-8}$ and a force threshold of $10^{-4}$ was used. A wavefunction cutoff (wfcut) of 50-60Ry and charge density (rhocut) cutoff of 560Ry were used for all pseudopotentials. Electron masses were kept at 300 a.u then increased to 450 after initial damped minimizations, to accelerate convergence of the initial structure as described in 4 to Figure 1
All calculations were done with periodic boundary conditions, and the Brillouin zone was sampled at the $\Gamma$ point. Throughout the zero-field damped dynamics and structure minimization steps, the size and volume of the simulation cell were continuously updated to allow for relaxation and minimization of stress of the crystal cell.

A thermostat was not applied nor were temperatures controlled. Test runs using thermostats between 200 and $600 \mathrm{~K}$ were performed: temperature effects, especially those on geometry were negligible in the present systems. We attribute this to the combination of a high melting temperature of the ionic matrix and the rigidity of the lattice due to high ionic charges.

Polarization was calculated using equation ?? in a static 0.001 a.u uniform field applied to the simulation cell using the method described by Umari and Pasquarello in Ref. ? The electronic polarization and BECs were calculated in the clamped-ion step of the procedure in Figure 5 (Step 8), where the electronic response is coupled to ionic positions. Further, the fully-relaxed static polarization was calculated by releasing the ionic clamps and allowing a full relaxation of the system while applying the static field (Step 9 in Figure 5).

\section{Results and Discussion}

The inclusion of silver atoms within the $\mathrm{MgO}$ matrix distorted the local structure (see Figure 1 for an example). The distortion, or 'stress', of the crystal structure only extended in general to the nearest neighbors of the inclusions (up to around 3 angstroms from the edge of the mNP). Sufficient $\mathrm{MgO}$ layers were added around the inclusion so that the NP-induced distortion did not extend to neighboring cells, this is seen in volume $\%$ of inclusion in simulation cell.

Densities of states. Calculated bandgaps of polarizable materials require careful consideration of the errors arising from the DFT formalism- especially when using LDA functional. The calculated bandgap is generally expected to be significantly lower than the corresponding bandgap of an experimental material with additional states introduced due to the inclusion. ${ }^{?}$ However, as Figures 7 ?? indicates, as well as from previous work in our group ??, much of the density of states in the vicinity of the Fermi level is arising from NP states, and is populated by the many NP electrons. Thus we expect DFT performance in these cases to mirror more closely its performance for nanoparticles (see references? ${ }^{\text {? }}$ ), than for bulk materials.

Dielectric response of the NC. Our previous work on the dielectric response of $\mathrm{Ag} / \mathrm{MgO} \mathrm{NCs}^{1}$ considered a single inclusion type, a "spherical" $\mathrm{Ag} 8 \mathrm{mNP}$. $\mathrm{An} \approx 30 \%$ increase in $\varepsilon_{r}$ was observed with an $\approx 4 \% \mathrm{mNP}$ volume ratio in the $\mathrm{Ag}_{8}$ case. Extension of the NP to rod-like and disk-like structures further enhances the dielectric response, both through increases in volume ratios, and via specific local field enhancements. $\varepsilon_{r}$ for $\mathrm{Ag} / \mathrm{MgO}$ NCs with rod-like and disk-like inclusions are presented in Table 1]. The data indicate that asymmetric inclusions bring on a dielectric enhancement that is, depending on orientation, even larger than expected based on volume ratios. For example, the multi-layer disks (disks 332, 432 and 442) exhibit more than $70 \%$ enhance- 

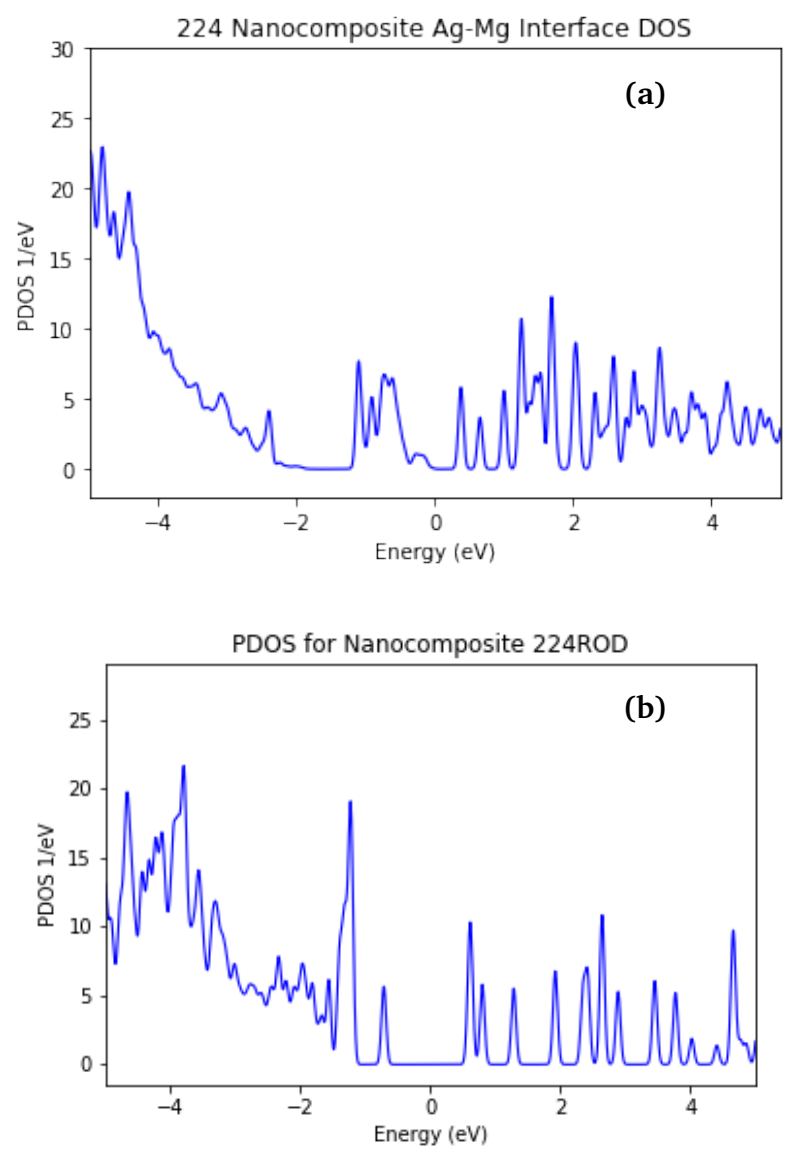

Fig. 7 Density Of States (DOS) for the Ag/MgO NC with the 224 rod-like inclusion. The full DOS is shown in (a), whereas (b) plots the inclusiononly projected DOS.

ment in $\varepsilon_{r}$ over the matrix permittivity, at similar volume loading (see Table 1). ADD DISC here tying to the next para.

\section{Local Polarization Fields}

The Born effective charges reflect the influence of the NP polarization on the charge distribution in the matrix ions. As a result, their interpolated plots provide effective maps of the local electrostatic field created by the NP's polarization in the matrix.

Figure 9 show interpolated maps for the NC with a 224 rodlike inclusion. Enhancement of the polarization field is seen in the matrix in regions adjacent to the NP poles, in the direction of the applied field. In the equatorial region a depolarization arises (red/yellow regions in BEC plots) where the effective ionic charge is less than in the absence of the external field. Whereas the enhancement is strongly localized to two layers of matrix ions at the interface, the (weaker) depolarization field is often extended over large regions of the cell.

Polarization perpendicular to the field ?? shows a much larger depolarization field around the nanoparticle that never decays to the normal bulk values (green) whereas the perpendicular depolarization area only affects the interfacial atoms.

Rod-like inclusions. All the rods considered here are similar in cross-section, with longer rods presenting additional atom planes. As rods increase in size, more electrons are available to
Table 1 Rod Nanoparticle Polarization

\begin{tabular}{|lrrrr|}
\hline Inclusion & $\mathrm{N}_{\text {atoms }}$ & Loading* & $\varepsilon_{\|}$ & $\varepsilon_{\perp}$ \\
\hline None & 0 & $4 \%$ & 9.8 & \\
CUBE 222 1 & 8 & $3.7 \%$ & 11.94 & \\
\hline ROD 223 & 12 & $4.2 \%$ & 12.94 & 11.03 \\
ROD 224 & 16 & $4.0 \%$ & 13.9 & 12.23 \\
ROD 225 & 20 & $3.9 \%$ & 14.87 & 12.79 \\
\hline DISK 332 & 18 & $4.3 \%$ & 14.97 & 11.84 \\
DISK 432 & 24 & $3.5 \%$ & 17.8 & $15 . *$ \\
DISK 442 & 32 & $4 \%$ & 19.61 & $* * *$ \\
DISK 241 & 8 & $4 \%$ & 12.8 & $11 . *$ \\
DISK 441 & 16 & $3.5 \%$ & 13.54 & 12.48 \\
* Calculated as a ratio of the number of atoms in the mNP to the total \\
number of atoms in the cell.
\end{tabular}

mobilize along the applied field and for a longer distance, inducing a higher polarization within the mNP than that seen for $\mathrm{Ag}_{8}$. This polarization leads to stronger local fields at the nanoparticlematrix interface. However, dielectric enhancement in general is significantly more complex than a simple correlation to the $\mathrm{mNP}$ length or the availability of polarizable electrons. This is evidenced by comparison of $\varepsilon_{\|}$for Rod224 and Disk332: Both mNPs have similar loading but different shapes. Disk332 shows a similar value to Rod224 with fewer atoms.

For the Rod-shaped inclusions, polarization along the z-axis ( $\varepsilon$ ) results in a greater increase in polarization from the bulk at 9.23 than the perpendicular $\varepsilon$. Disk nanoparticles show similar effects with an exponential increase in $\varepsilon$ compared to their rod counterparts. Disk442 is twice the $\mathrm{x}, \mathrm{y}$ dimensions of Rod223 yet has a polarization increase of more than twice in comparison.

We suspect the there may be a threshold at which this exponential growth changes as the inclusion behaves more metallic however computational constraints limit us to this size.

Disk-like inclusions The disk-like Nanoparticles show the direction and shape effects to an increased degree. The 3dimensional mNPS of (332,432 and 442) all showed a higher polarization than similarly sized rods likely due to more moving electrons with greater space to move in the nanoparticle.

The 2-Dimensional nanoparticles (421 and 441) show a decrease in polarization when compared to the bulk in both directions. Since the inclusion electrons are not free to move in the z-direction they do not contribute to the increased average polarization.

\section{Induced Fields and Born Effective Charge}

In order to visualize the forces in these different NCs, 9 shows a smeared BEC plot of normalized born charges. These show that the forces contributing to the increased $\left(\varepsilon_{\text {Parallel }}\right)$ or decreased $\left(\varepsilon_{\text {Perpendicular }}\right)$ are mainly due to forces felt by atoms at the matrixnanoparticle interface. As the electrons in the silver mNP polarize, they move to the interface/matrix and increase the permittivity (blue) or decrease (yellow/orange) compared to the rest of the matrix (green). The shape of the nanoparticle influences the shape and reach of the induced polarization. Previous computational investigation into this showed that a significant increase in silver d-electron polarization as they couple to the oxygen pband $^{1}$. Mazimally-localized wannier functions were attempted 
however nanoparticles larger than 8 atoms were unable to converge due to high delocalization and movement of wannier centers 38

9 plot a) shows that the nanoparticles exhibit an increase in 'force' along the direction of polarization due to increased charge density. This raises the overall dielectric constant of the cell. Similarly the perpendicular polarization plot b) shows the opposite happens. The BEC plots resemble an induced field on the nanoparticle with moving electrons throughout the simulation creating areas of higher and lower force. This was supported in the calculation with an oscillating value of forces as the electrons continue to move even after the ions have been clamped.

When a nanoparticle is oriented parallel to the applied field we see the 'reach' of induced field is further physically and also stronger. For perpendicular polarization, the BEC converges to $+2,-2$ values of matrix in a very short distance, meaning a smaller field, smaller effect therefore lower avg dielectric constant.

** I should add more here on how the interfacial effects of these nanoparticles are the most important, as these nanoparticles are encapsulated by the matrix we can take advantage of and isolate this interfacial effect $* *$

\section{Conclusions}

From this work we have built an understanding of how polarization of molecular-scale inclusions affect the induced field or induced charges on a matrix metal-oxide dielectric. Molecular scale metal inclusions of silver in a magnesium oxide matrix have shown to increase the local dielectric capacity depending on the shape, size and orientation to the external field. As the inclusions are small enough to behave as molecules and not bulk metal, calculation of electron dynamics and Born changes gives us a picture of induced fields around said inclusions. From polarization of mNPs arises induced fields at the matrix-inclusion interface. the nature of the induced field depends on the electron dynamics (charge dynamics? word?) as the metal atom polarizes. Increasing the atoms aligned to the applied field increases the charge density in the interface - larger areas of increased effective polarizability. The same is true for the inverse when inclusions are not aligned with the applied field. Disk shaped mNPs particularly suffer from this issue as we see severe screening rings around the inclusion the interacts with neighboring inclusions.

Future work would expand this into other matrix materials such as $\mathrm{SiO}_{2}$ and $\mathrm{HfO}_{2}$ among others. Additionally, simulation of other transition metal inclusions of industrial use such as $\mathrm{Cu}$, Co, Fe etc... and interaction between these would build a more robust understanding of molecular scale NC materials and 'quantum' polarization effects...

- See a certain amount of interaction in these induced fieldsalways perpendicular to the direction of polarization due to distortion of internal field on mNP

- is this limiting the 'reach' of polarization.. as in the local effects cannot extend beyond these destructive bands-

- sometimes this perpendicular response in the matrix dominates the overall polarization and the dielectric constant cal- culated is lower than for pure matrix

1. close control on direction must be maintained,

2. \%loading is not the only thing, aggregation and separation Is very important,

3. if direction effects are important in a crystal without directional polarization, this adds a layer of complexity of crystal structures.

4. induced fields in nanoscale composites is a way to understand quantum materials behaviour as materials get smaller and more precise.

\section{Conflicts of interest}

\section{Acknowledgements}

We would like to acknowledge ?? uvic, Paci group members? westgrid/computecanada but not the sysadmins lol 
Journal Name, [year], [vol.], $1-10$ | 7 


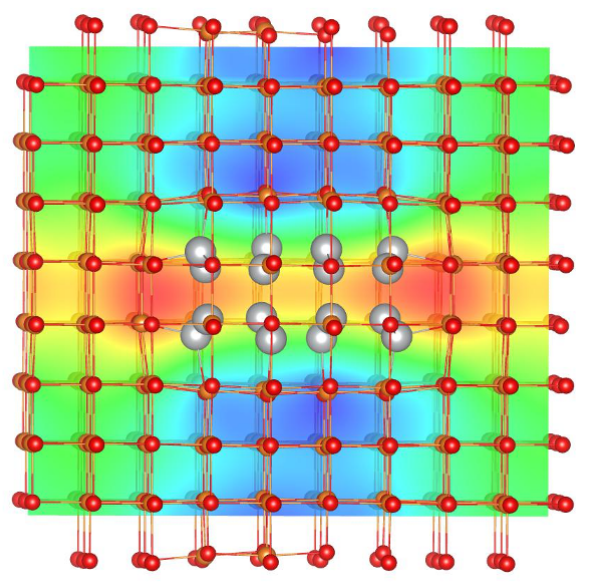

(a)

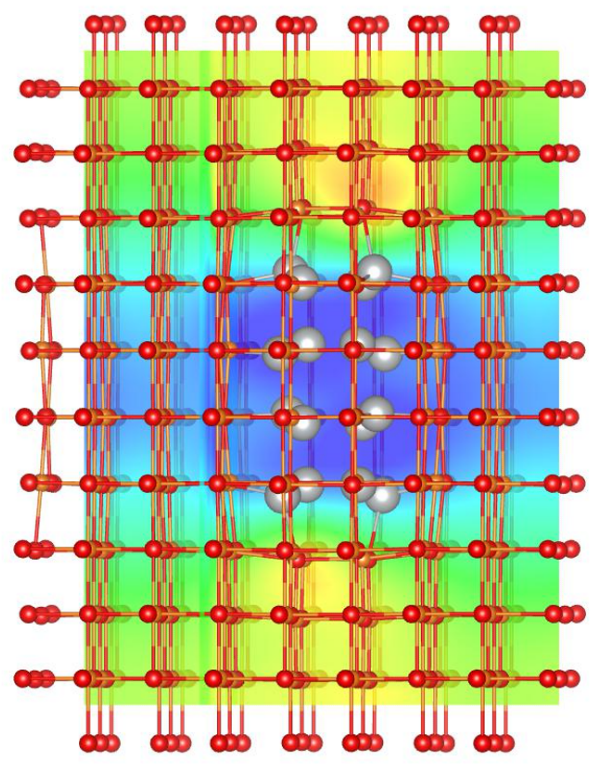

(c)

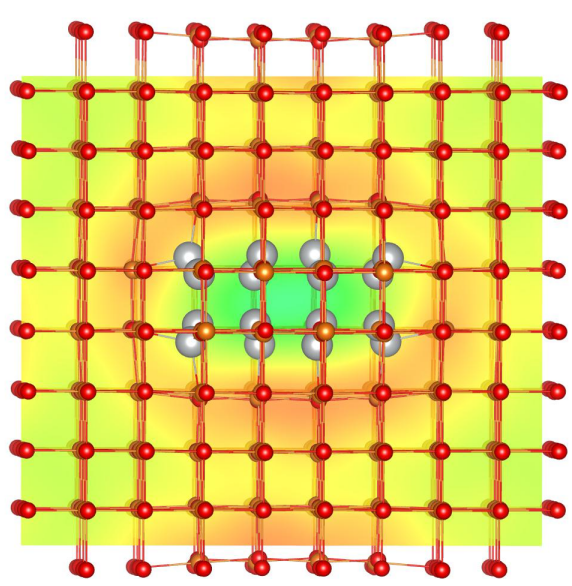

(b)

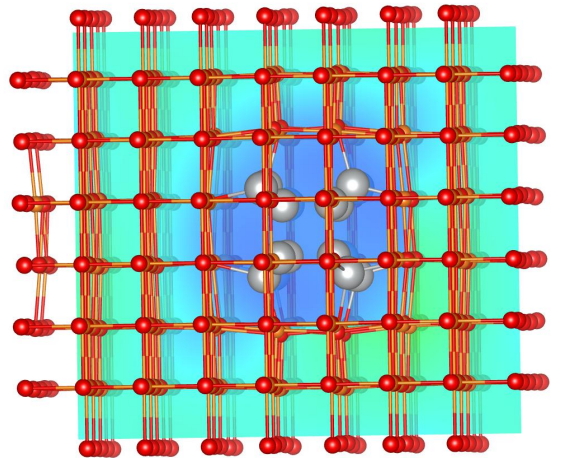

(d)

Fig. 9 BEC plots showing areas of increased (blue) and decreased (red) polarization compared to bulk matrix values. Polarization is always in the z-direction - (a) shows the xz plane for a 224 rod inclusion perpendicular polarization, (b) shows xy plane of the same nanoparticle. 'Perpendicular' polarization means the applied field is perpendicular to the longest axis of the inclusion and exhibits 'depolarization' rings - areas of lower polarization compared to matrix. (c) Shows a 224 rod parallel polarization in the xz plane, and (d) shows the same in xy plane. 
Journal Name, [year], [vol.], $1-10$ | 9 


\section{Notes and references}

1 D. J. Hally and I. Paci, Nanoscale, 2018, 10, 9583-9593.

2 L. Kong, S. Li, T. Zhang, J. Zhai, F. Boey and J. Ma, Progress in Materials Science, 2010, 55, 840-893.

3 G. Subramanyam, M. W. Cole, N. X. Sun, T. S. Kalkur, N. M. Sbrockey, G. S. Tompa, X. Guo, C. Chen, S. P. Alpay, G. A. Rossetti, K. Dayal, L.-Q. Chen and D. G. Schlom, Journal of Applied Physics, 2013, 114, 191301.

4 M. R. Lukatskaya, B. Dunn and Y. Gogotsi, Nature Communications, 2016, 7, 1-13.

5 D. Shamiryan, T. Abell, F. Iacopi and K. Maex, Materials Today, 2004, 7, 34-39.

6 X. Yang, Z. Zhou, T. Nan, Y. Gao, G. M. Yang, M. Liu and N. X. Sun, Journal of Materials Chemistry C, 2016, 4, 234-243.

7 J. Robertson, The European Physical Journal Applied Physics, 2004, 28, 265-291.

8 F. Balestra and G. Ghibaudo, Semiconductor Science and Technology, 2017, 32, 023002.

9 S. B. Zhang, S.-H. Wei and A. Zunger, Phys. Rev. B, 2001, 63, 075205.

10 N. Umezawa, K. Shiraishi, T. Ohno, H. Watanabe, T. Chikyow, K. Torii, K. Yamabe, K. Yamada, H. Kitajima and T. Arikado, Applied Physics Letters, 2005, 86, 143507.

11 H. Jiang, K. S. Moon, Y. Li and C. P. Wong, Chemistry of Materials, 2006, 18, 2969-2973.

12 T. Pettong, P. Iamprasertkun, A. Krittayavathananon, P. Sukha, P. Sirisinudomkit, A. Seubsai, M. Chareonpanich, P. Kongkachuichay, J. Limtrakul and M. Sawangphruk, ACS Applied Materials \& Interfaces, 2016, 8, 34045-34053.

13 J. Robertson and R. M. Wallace, Materials Science and Engineering: R: Reports, 2015, 88, 1-41.

14 C. L. Jia, S. B. Mi, K. Urban, I. Vrejoiu, M. Alexe and D. Hesse, Phys. Rev. Lett., 2009, 102, 117601.

15 N. Shi and R. Ramprasad, Phys. Rev. B, 2006, 74, 045318.

16 W. P. Halperin, Reviews of Modern Physics, 1986, 58, 533-606.

17 J. Y. Li, L. Zhang and S. Ducharme, Applied Physics Letters, 2007, 90, 132901.

18 V. Kochergin, V. Zaporojtchenko, H. Takele, F. Faupel and H. Föll, Journal of Applied Physics, 2007, 101, 024302.

19 G. Grzela, D. Hourlier and J. Gómez Rivas, Phys. Rev. B, 2012, 86, 045305.

20 F. Aryasetiawan, O. Gunnarsson, M. Knupfer and J. Fink, Physical Review B, 1994, 50, 7311-7321.

21 L. Lajaunie, F. Boucher, R. Dessapt and P. Moreau, Phys. Rev. B, 2013, 88, 115141.

22 L. L. Canfield, PhD thesis, 1992.

23 N. Marzari and D. Vanderbilt, Physical Review B - Condensed Matter and Materials Physics, 1997, 56, 12847-12865.

24 N. A. Spaldin, Journal of Solid State Chemistry, 2012, 195, 2-10.
25 L. Yan, C. M. Lopez, R. P. Shrestha, E. A. Irene, A. A. Suvorova and M. Saunders, Applied Physics Letters, 2006, 88, 142901.

26 A. Posadas, F. J. Walker, C. H. Ahn, T. L. Goodrich, Z. Cai and K. S. Ziemer, Applied Physics Letters, 2008, 92, 233511.

27 X. Liu, D. Mazumdar, W. Shen, B. D. Schrag and G. Xiao, Applied Physics Letters, 2006, 89, 023504.

28 A. Hirohata, K. Yamada, Y. Nakatani, I.-L. Prejbeanu, B. Diény, P. Pirro and B. Hillebrands, Journal of Magnetism and Magnetic Materials, 2020, 509, 166711.

29 M. D. Hanwell, D. E. Curtis, D. C. Lonie, T. Vandermeersch, E. Zurek and G. R. Hutchison, Journal of Cheminformatics, 2012, 4, year.

30 R. Car and M. Parrinello, Physical Review Letters, 1985, 55, 2471-2474.

31 P. Giannozzi, S. Baroni, N. Bonini, M. Calandra, R. Car, C. Cavazzoni, D. Ceresoli, G. L. Chiarotti, M. Cococcioni, I. Dabo, A. D. Corso, S. de Gironcoli, S. Fabris, G. Fratesi, R. Gebauer, U. Gerstmann, C. Gougoussis, A. Kokalj, M. Lazzeri, L. Martin-Samos, N. Marzari, F. Mauri, R. Mazzarello, S. Paolini, A. Pasquarello, L. Paulatto, C. Sbraccia, S. Scandolo, G. Sclauzero, A. P. Seitsonen, A. Smogunov, P. Umari and R. M. Wentzcovitch, Journal of Physics: Condensed Matter, 2009, 21, 395502.

32 P. Umari and A. Pasquarello, Phys. Rev. Lett., 2002, 89, 157602.

33 N. Wiser, Phys. Rev., 1963, 129, 62-69.

34 Y. Li, B. Xiao, L. Sun, Y. Gao and Y. Cheng, Journal of Alloys and Compounds, 2017, 692, 713-719.

35 M. van Setten, M. Giantomassi, E. Bousquet, M. Verstraete, D. Hamann, X. Gonze and G.-M. Rignanese, Computer Physics Communications, 2018, 226, 39-54.

36 A. D. Corso, Computational Materials Science, 2014, 95, 337350.

37 K. Lejaeghere, G. Bihlmayer, T. Bjorkman, P. Blaha, S. Blugel, V. Blum, D. Caliste, I. E. Castelli, S. J. Clark, A. D. Corso, S. de Gironcoli, T. Deutsch, J. K. Dewhurst, I. D. Marco, C. Draxl, M. D. ak, O. Eriksson, J. A. Flores-Livas, K. F. Garrity, L. Genovese, P. Giannozzi, M. Giantomassi, S. Goedecker, X. Gonze, O. Granas, E. K. U. Gross, A. Gulans, F. Gygi, D. R. Hamann, P. J. Hasnip, N. A. W. Holzwarth, D. I. an, D. B. Jochym, F. Jollet, D. Jones, G. Kresse, K. Koepernik, E. Kucukbenli, Y. O. Kvashnin, I. L. M. Locht, S. Lubeck, M. Marsman, N. Marzari, U. Nitzsche, L. Nordstrom, T. Ozaki, L. Paulatto, C. J. Pickard, W. Poelmans, M. I. J. Probert, K. Refson, M. Richter, G.-M. Rignanese, S. Saha, M. Scheffler, M. Schlipf, K. Schwarz, S. Sharma, F. Tavazza, P. Thunstrom, A. Tkatchenko, M. Torrent, D. Vanderbilt, M. J. van Setten, V. V. Speybroeck, J. M. Wills, J. R. Yates, G.-X. Zhang and S. Cottenier, Science, 2016, 351, aad3000.

38 I. Souza, T. Wilkens and R. M. Martin, Physical Review B, 2000, 62, 1666-1683. 